\title{
Communication \\ Phase Compensation Method in OPA System Based on the Linear Electro-Optic Effect
}

\author{
Shuaishuai Yang ${ }^{1,2}$ (1) and Dean Liu ${ }^{1, *}$ \\ 1 Key Laboratory of High Power Laser and Physics, Shanghai Institute of Optics and Fine Mechanics, \\ Chinese Academy of Sciences, Shanghai 201800, China; ssyang@siom.ac.cn \\ 2 Center of Materials Science and Optoelectronics Engineering, University of Chinese Academy of Sciences, \\ Beijing 100049, China \\ * Correspondence: Liudean@siom.ac.cn
}

check for updates

Citation: Yang, S.; Liu, D. Phase Compensation Method in OPA System Based on the Linear Electro-Optic Effect. Photonics 2021, 8, 126. https://doi.org/10.3390/ photonics 8040126

Received: 16 March 2021

Accepted: 15 April 2021

Published: 17 April 2021

Publisher's Note: MDPI stays neutral with regard to jurisdictional claims in published maps and institutional affiliations.

Copyright: (c) 2021 by the authors. Licensee MDPI, Basel, Switzerland. This article is an open access article distributed under the terms and conditions of the Creative Commons Attribution (CC BY) license (https:// creativecommons.org/licenses/by/ $4.0 /)$.

\begin{abstract}
Factors such as mechanical deformation and temperature changes lead to phase mismatch in optical parametric amplification systems, impacting energy stability. A phase compensation method via the linear electro-optic effect can overcome this limitation. Phase mismatch compensation characteristics were simulated via the linear electro-optic effect in 70\%-deuterated DKDP and 95\%deuterated DKDP. This method was applied to OPA systems to verify its feasibility. The results show that the temperature acceptance bandwidth of 70\%-deuterated DKDP and 95\%-deuterated DKDP can be $\sim 1.75$ and $\sim 2$ times larger, respectively, than that of the OPA without compensation. Moreover, the angle acceptance bandwidth of 70\%-deuterated DKDP and 95\%-deuterated DKDP can be $\sim 2$ times larger than that of the OPA without compensation. The abovementioned method can facilitate the compensation of phase mismatch within a range and can be widely used in OPA and optical parametric chirped pulse amplification systems to improve laser stability.
\end{abstract}

Keywords: OPA; phase compensation; linear electro-optic effect; DKDP

\section{Introduction}

Since the optical parametric chirped pulse amplification (OPCPA) technology has been proposed [1], further developments have been achieved in the field of high-power lasers. For example, assorted OPCPA systems have been designed and constructed [2,3]. A quintessential system is the SG-II 5-PW laser facility [4], containing three optical parametric amplification (OPA) stages. The final energy of its compressed pulses can reach $150 \mathrm{~J}$ with a pulse duration of $30 \mathrm{fs}$, corresponding to a peak power of $5 \mathrm{PW}$. Indeed, BBO crystals are used in a preamplifier because of their high nonlinear coefficient and optical aperture. Moreover, amplifiers of $\mathrm{LBO}$ and $\mathrm{YCOB}$ crystals are generally used as main amplifiers at later stages [5-8]. LBO, YCOB, KDP, and DKDP nonlinear crystals are the best candidates for OPCPA systems [9-11]. LBO and YCOB crystals have strong nonlinearity; however, when subject to the current crystal growth technology, only crystals with diameters less than 150 and $70 \mathrm{~mm}$, respectively, can be obtained. In a typical PW-level high-power laser facility, the beam diameter of a last-stage amplifier is usually more than $50 \mathrm{~mm}$, and the diameter requirements for a 5-PW-level or 10-PW-level laser facility are more than $50 \mathrm{~mm}$. Despite the fact that KDP and DKDP crystals display weak nonlinearity, they are the only crystals that can be grown to an aperture of $300 \mathrm{~mm}$ or more [12]. Therefore, they are promising nonlinear crystals used in main amplifiers in OPCPA laser systems. For an optical parametric amplifier pumped at $527 \mathrm{~nm}$, KDP and DKDP crystals can achieve wide gain bandwidths at a wavelength of $910 \mathrm{~nm}$. The Russian XCELS facility is an OPCPA system based on DKDP at the wavelength of $910 \mathrm{~nm}$. Highly deuterated DKDP crystals can achieve a broad parametric bandwidth at $800 \mathrm{~nm}$ [13]. Especially in 95\%-deuterated DKDP, parametric bandwidths of $800 \mathrm{~nm}$ wavelength can reach $>35$ and $>50 \mathrm{~nm}$ with pump pulse at 532 and $527 \mathrm{~nm}$, respectively. However, the idlers in 
some OPA systems are in the absorption region of the DKDP crystal, and the DKDP is a temperature-sensitive crystal. Especially in some high-repetition ultra-intense laser systems, the temperature of DKDP crystals gradually rises, resulting in phase mismatch. Moreover, the mechanical deformation can also cause phase mismatch in large laser systems. OPA progress conversion efficiency varies with the phase mismatch. We present a phase mismatch compensation method to minimize the effects of temperature and mechanical deformation. DKDP crystals are used as nonlinear and electro-optic crystals in the OPA system. The electro-optic effect can change the refractive index to compensate for the phase mismatch caused by temperature change or mechanical deformation [14]. In this study, a $1064 \mathrm{~nm}$ OPA system is adopted to verify the feasibility of this method. This method has universal applicability in OPA systems at different wavelengths, and it can be used to improve the stability of OPCPA systems.

\section{Phase Mismatch Compensation Simulation}

The linear electro-optic effect can change the refractive index of a crystal; therefore, phase mismatch can be compensated via the linear electro-optic effect. In particular, DKDP crystals have sensitive electro-optic effects because of a high value of the electro-optic coefficient. Measurements of the electro-optic coefficient of DKDP have been previously reported. The relationship between the deuterium content $(D)$ and linear electro-optic coefficient $\left(\gamma_{63}\right)$ is $\gamma_{63}=-9.789-16.53 \mathrm{D}$ [15]. According to the principle of linear electro-optic effect and DKDP crystal refractive index ellipsoid equation, the new principle polarization axes $\mathrm{x}^{\prime}$ and $\mathrm{y}^{\prime}$ are rotated $45^{\circ}$ around the $\mathrm{z}$-axis from the original polarization axes with a constant electric field applied to DKDP along the z-direction, and thus [16]

$$
\begin{gathered}
n_{x^{\prime}}=n_{x}+\frac{1}{2} n_{x}^{3} \gamma_{63} E_{z}, \\
n_{y^{\prime}}=n_{y}-\frac{1}{2} n_{y}^{3} \gamma_{63} E_{z}, \\
n_{z^{\prime}}=n_{z},
\end{gathered}
$$

where the $n_{x}$ and $n_{y}$ are equal to ordinary refractive index $n_{0}, n_{z}$ is the extraordinary refractive index $n_{e}, \gamma_{63}$ is the electro-optic coefficient, and $E_{z}$ is electric field intensity (given by $\mathrm{V} / \mathrm{d}$, where $d$ is thickness along the z-direction). The temperature behavior of the refractive index is described using $d n / d T$. Refractive index with respect to temperature is given as $d n_{o} / d T \sim-3 \times 10^{-5} \mathrm{~T}^{-1}$ and $d n_{e} / d T \sim-2 \times 10^{-5} \mathrm{~T}^{-1}$ [17].

In this study, the 70\%-deuterated DKDP and 95\%-deuterated DKDP were selected as the research objects. Since the injected signal energy is relatively high, and in order to display the acceptable bandwidth, we define the conversion efficiency as $\Delta E_{\text {signal }} / E_{\text {pump }}$. $\Delta E_{\text {signal }}$ and $E_{\text {pump }}$ respectively represent the increased energy of the signal and the input pump energy. To compare the effects on OPA conversion efficiency before and after compensation, the conversion efficiencies in the corresponding case were simulated and are shown in Figure 1. The crystal size is $20 \mathrm{~mm} \times 20 \mathrm{~mm} \times 30 \mathrm{~mm}$ (crystal length is $30 \mathrm{~mm}$ ), the pump intensity and signal intensity are $\sim 2$ and $\sim 1 \mathrm{GW} / \mathrm{cm}^{2}$, respectively. The wavelengths of the pump laser and signal laser are $532 \mathrm{~nm}$ and $1064 \mathrm{~nm}$, respectively. In the process of OPA based on 70\%-deuterated DKDP crystal, the phase-matching angle $(\theta)$ and non-collinear angle $(\alpha)$ are 38.20 and $0.898^{\circ}$, respectively. For the $95 \%$-deuterated DKDP crystal, the phase-matching angle $(\theta)$ and non-collinear angle $(\alpha)$ are 36.95 and $0.928^{\circ}$, respectively. Figure 1a,b shows the temperature-dependent OPA conversion efficiencies of $70 \%$-deuterated and 95\%-deuterated DKDP. After the temperature changes, an optimal voltage value is selected to compensate for the phase mismatch. The voltage value of $10 \mathrm{kV}$ can compensate for the phase mismatch caused by a temperature change of $\sim 5$ and $\sim 6{ }^{\circ} \mathrm{C}$ for $70 \%$-deuterated and $95 \%$-deuterated DKDP, respectively. Figure $2 \mathrm{a}$, b shows the angle-dependent OPA conversion efficiencies of 70\%-deuterated and 95\%-deuterated DKDP. The phase mismatch is also compensated using an optimal voltage value. The 
voltage value of $10 \mathrm{kV}$ can compensate for a phase mismatch caused by $\sim 0.02$ and $\sim 0.03^{\circ}$ angle changes for $70 \%$-deuterated and $95 \%$-deuterated DKDP, respectively.
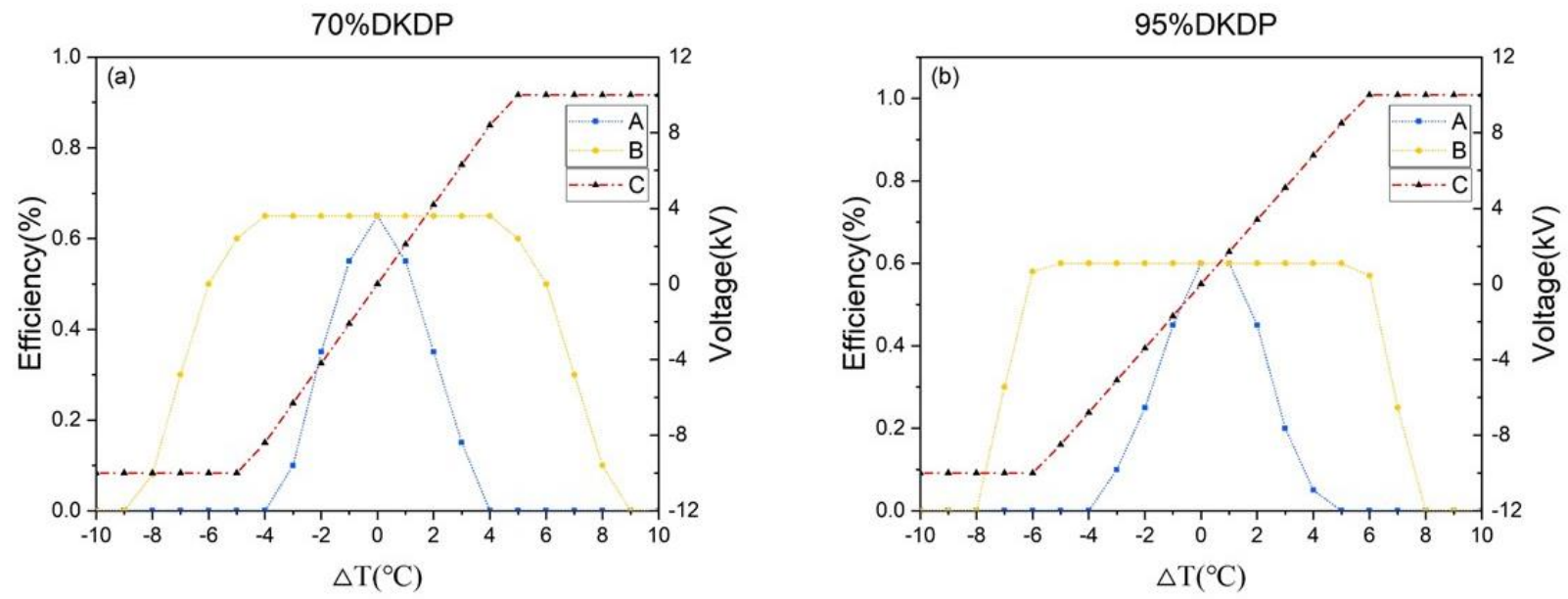

Figure 1. Calculated OPA efficiencies obtained via (a) 70\%- and (b) 95\%-deuterated DKDP versus temperature and compensation voltage. Line A represents the OPA efficiency without compensation, Line B indicates OPA efficiency where the phase mismatch is compensated, and Line $C$ represents the compensation voltage.
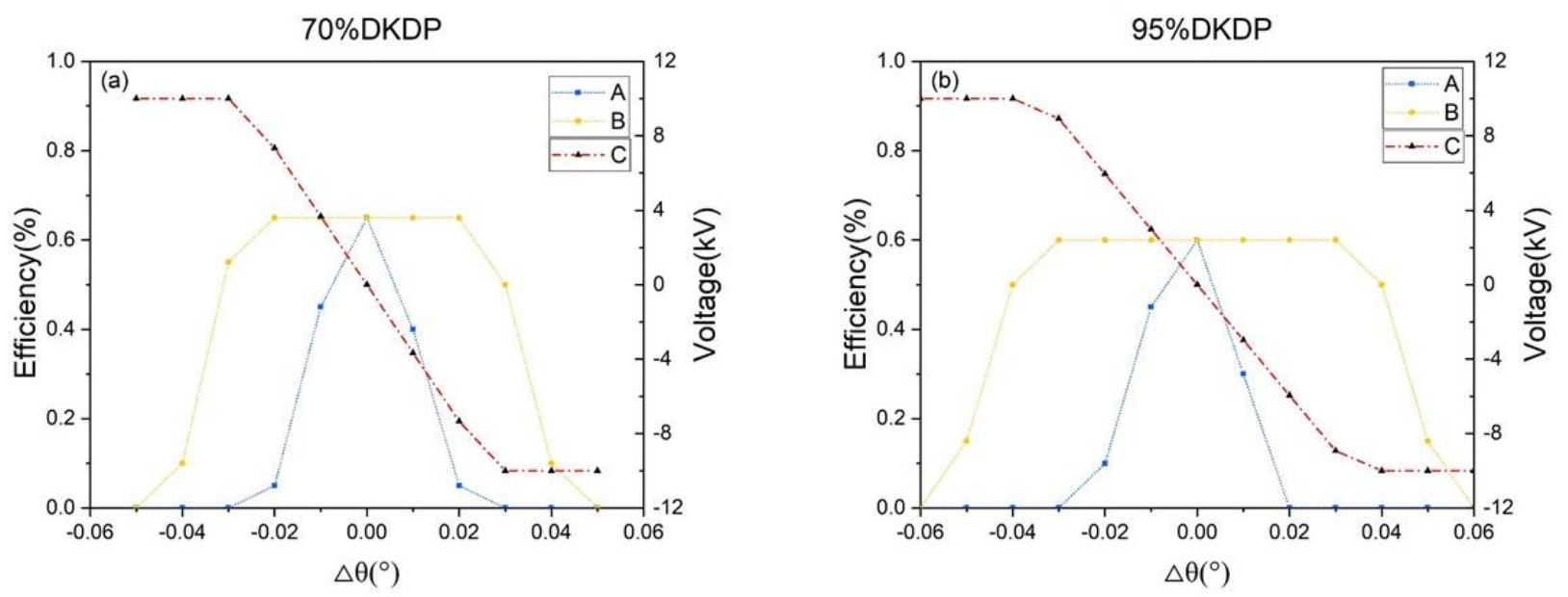

Figure 2. Calculated OPA efficiencies obtained via (a) 70\%- and (b) 95\%-deuterated DKDP versus angle and compensation voltage. Line A represents the OPA efficiency without compensation, Line B indicates OPA efficiency where the phase mismatch is compensated, and Line $C$ represents the compensation voltage.

Figure 3 shows the phase mismatch compensation voltage versus the temperature and angle changes. Compared with the 95\%-deuterated DKDP, the 70\%-deuterated DKDP requires a higher voltage value to compensate for phase mismatch. It can be seen from Equation (1) that the change of refractive index is

$$
\Delta n=\frac{1}{2} n_{o}^{3} \gamma_{63} E .
$$



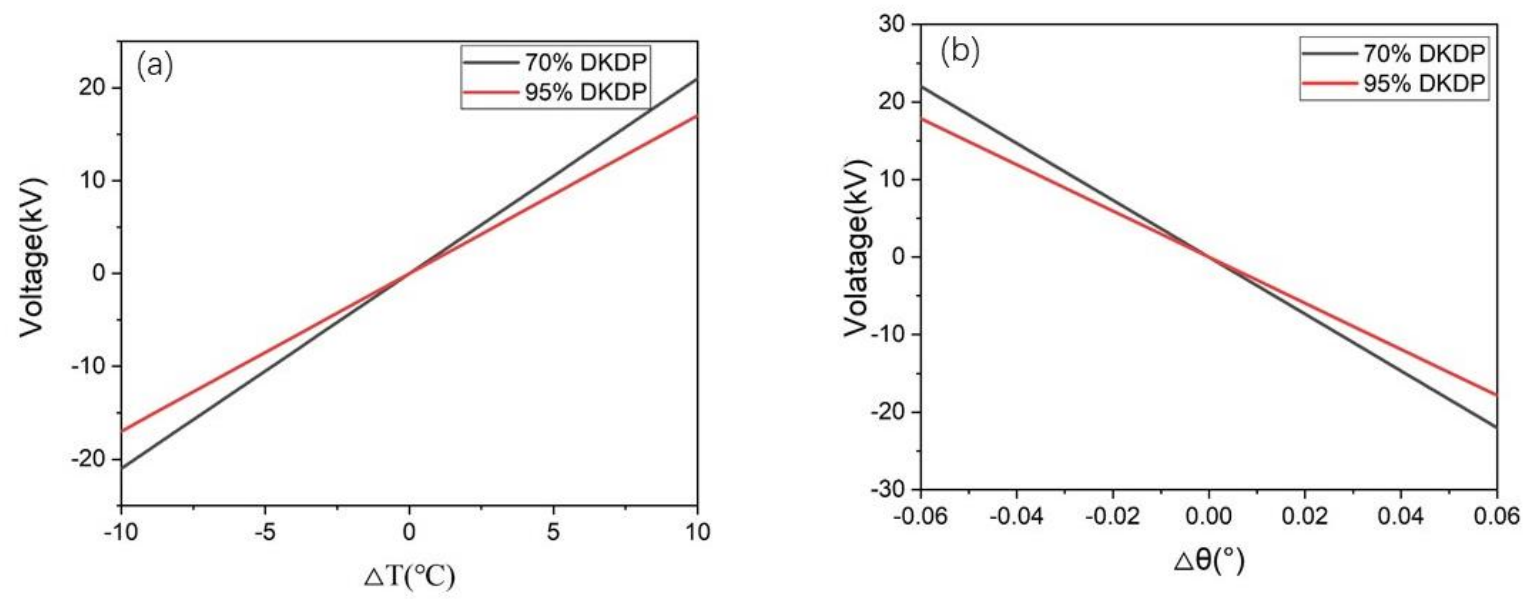

Figure 3. (a) Calculated phase mismatch compensation voltage versus temperature; (b) calculated phase mismatch compensation voltage versus angle.

Here, we define $\xi=n_{o}^{3} \gamma_{63}$. According to previous works, the $\xi$ values of $70 \%$ deuterated DKDP and 95\%-deuterated DKDP are $\sim 71.10 \mathrm{pm} / \mathrm{V}$ and $\sim 84.82 \mathrm{pm} / \mathrm{V}$, respectively. Therefore, the $95 \%$-deuterated DKDP requires a lower voltage for phase mismatch compensation.

\section{Experimental Setup and Results}

The phase compensation method was applied to an OPA system to verify the feasibility of the method. The experimental setup is shown in Figure 4.

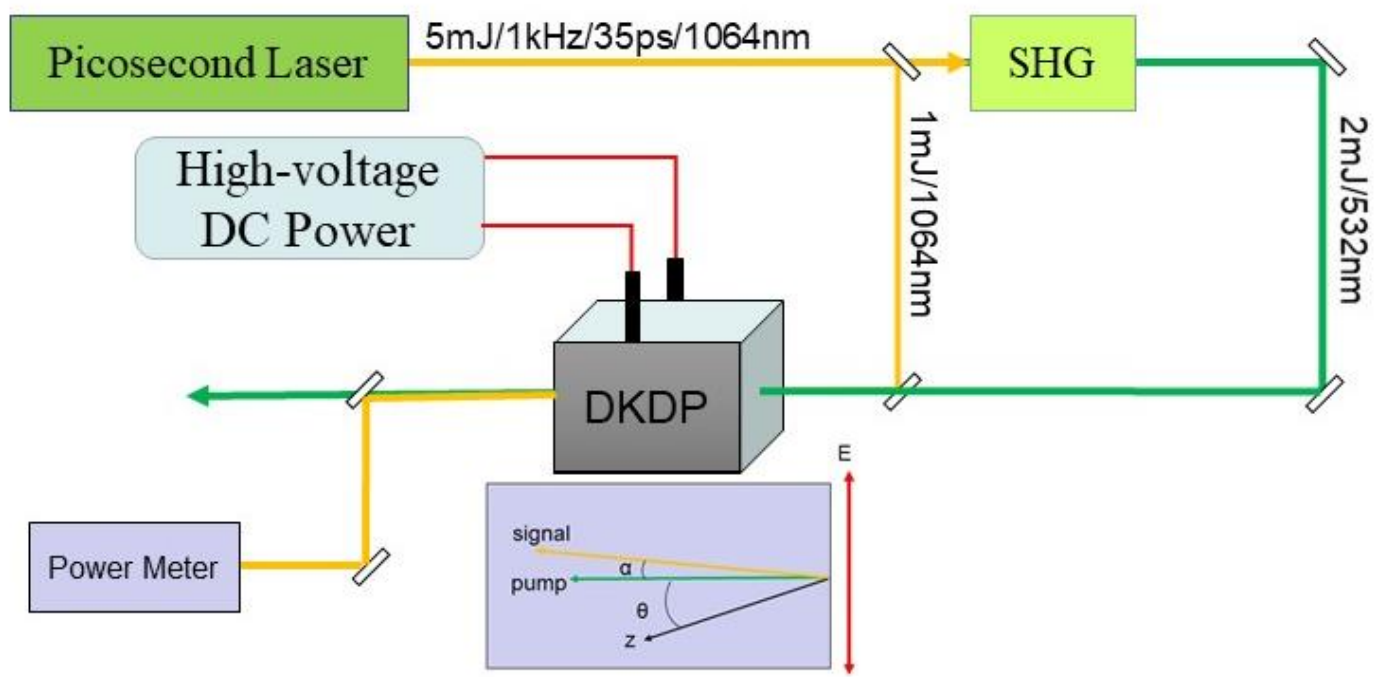

Figure 4. Phase compensation experimental setup. SHG: LBO crystal with the size of $4 \mathrm{~mm} \times 4 \mathrm{~mm} \times 15 \mathrm{~mm}$; yellow line represents pulses at $1064 \mathrm{~nm}$; green line represents pulses at $532 \mathrm{~nm}$.

The pump source was a $1 \mathrm{kHz}$ Nd:YAG picosecond laser exhibiting a pulse energy of $>5 \mathrm{~mJ}$ at 1064 with a pulse duration of $\sim 35 \mathrm{ps}$. Since the repetition rate of the laser was $1 \mathrm{kHz}$, the corresponding single pulse energy could be calculated by measuring the laser power with a power meter. The beam diameter of the laser was $\sim 2 \mathrm{~mm}$ $\left(1 / \mathrm{e}^{2}\right.$ level). A small fraction $(\sim 1 \mathrm{~mJ})$ was used as signal pulses (corresponding peak power was $\sim 1 \mathrm{GW} / \mathrm{cm}^{2}$ ). The remaining part was subsequently frequency-doubled and delivered to DKDP amplifiers. The pump energy was $2 \mathrm{~mJ}$ at $532 \mathrm{~nm}$ (corresponding peak power was $\sim 2 \mathrm{GW} / \mathrm{cm}^{2}$ ). The $70 \%$-deuterated DKDP crystal used in the system was cut into the size of $20 \mathrm{~mm} \times 20 \mathrm{~mm} \times 30 \mathrm{~mm}$ (crystal length is $30 \mathrm{~mm}$ ) for critically phase-matched 
Type I $\left(\theta=38.2^{\circ}, \alpha=0.898^{\circ}\right)$. After the $70 \%$-deuterated DKDP amplifier, the output energy was $1.8 \mathrm{~mJ}$. The $95 \%$-deuterated DKDP crystal used in the system was cut into the size of $20 \mathrm{~mm} \times 20 \mathrm{~mm} \times 30 \mathrm{~mm}$ (crystal length is $30 \mathrm{~mm}$ ) for critically phase-matched Type I $\left(\theta=36.95^{\circ}, \alpha=0.928^{\circ}\right)$. After the $95 \%$-deuterated DKDP amplifier, the output energy was $\sim 1.7 \mathrm{~mJ}$. The direction of the applied electric field in the experiment was along the cutting direction of DKDP (Figure 3); thus,

$$
E_{z}=E \cdot \sin \theta
$$

where $E$ and $E_{z}$ are the applied electric field and the electric field along the z-axis, respectively. $\theta$ is the phase-matching angle of DKDP. The maximum output voltage for the DC voltage generator is $\sim 10 \mathrm{kV}$. Due to $\gamma_{63} \gg \gamma_{52}$ and $\gamma_{63} \gg \gamma_{41}$, we can ignore the electro-optic effects caused by the electric fields in the $\mathrm{x}$ and $\mathrm{y}$ directions. Therefore, the refractive index can be described by formulas (1)-(3).

To study the temperature-sensitive characteristics of DKDP, a hot air blower and a thermoelectric cooler (TEC) were used to change and stabilize the crystal temperature. The hot air blower can quickly help the crystal change temperature. A TEC was placed on the bottom of the crystal, and the temperature sensor was placed on the top of the crystal, allowing the TEC to be heated or cooled automatically by measuring the current crystal temperature. After the temperature changed, an optimal DC voltage was applied to the DKDP to compensate for the phase mismatch caused by the temperature changes.

Figure 5 shows the OPA efficiencies obtained via DKDP versus temperature and compensation voltage. The temperature compensation ranges were -4 to $+4{ }^{\circ} \mathrm{C}$ and -5 to $+5{ }^{\circ} \mathrm{C}$ for $70 \%$-deuterated DKDP and $95 \%$-deuterated DKDP, respectively. Moreover, with the temperature change of $1{ }^{\circ} \mathrm{C}$, the compensation voltage values required for $70 \%$ deuterated DKDP and 95\%-deuterated DKDP were $\sim 2.3$ and $\sim 1.9 \mathrm{kV}$, respectively. The acceptance bandwidth can be defined by the full width at half maximum (FWHM). The temperature acceptance bandwidth (FWHM) of 70\%-deuterated DKDP and 95\%-deuterated DKDP can reach $\sim 14$ and $\sim 16^{\circ} \mathrm{C}$, respectively, which is $\sim 1.75$ times and $\sim 2$ times larger than that of OPA without compensation.
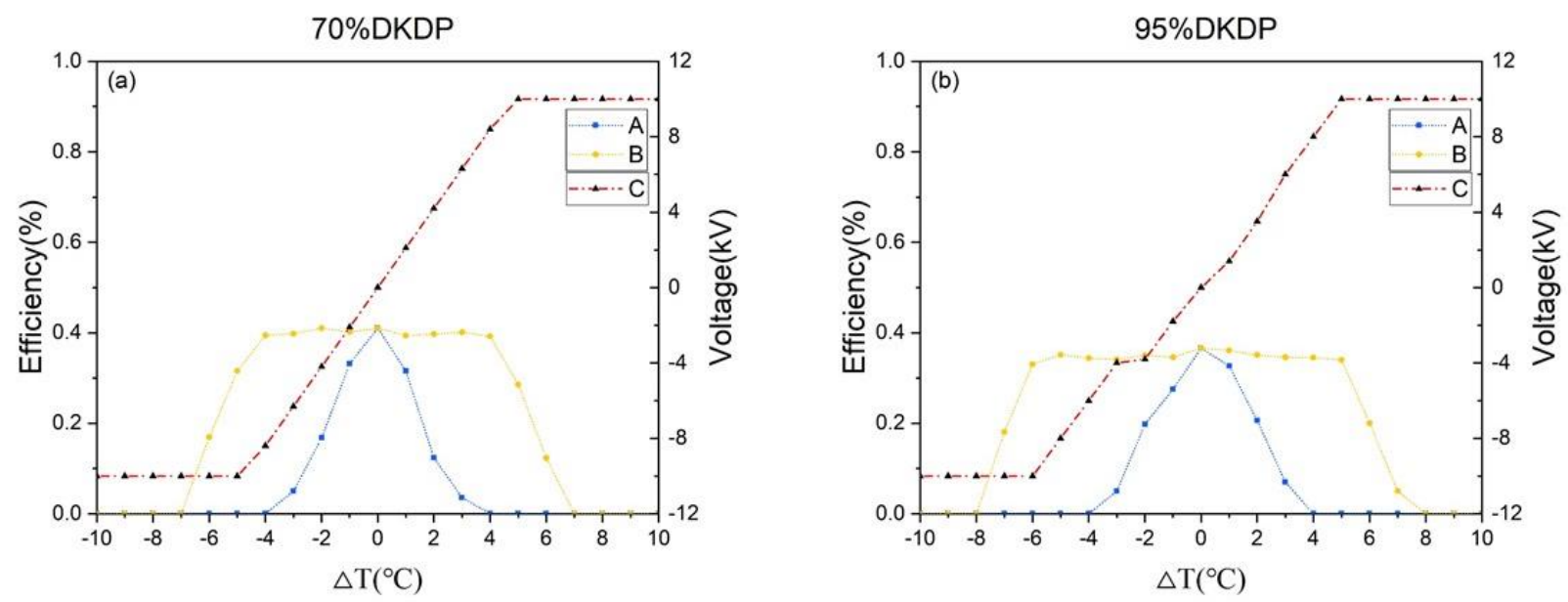

Figure 5. Experimental OPA efficiencies obtained via (a) 70\%- and (b) 95\%-deuterated DKDP versus temperature and compensation voltage. Line A represents the OPA efficiency without compensation, Line B indicates OPA efficiency where the phase mismatch is compensated, and Line $C$ represents the compensation voltage.

To study the angle-sensitive characteristics of DKDP, a high-precision rotation stage was used to change the phase-matching angle. The high-precision rotation stage provided precise, fine rotation for the DKDP crystal. The phase-matching angle could be smoothly and continuously rotated by the rotation stage, and then a corresponding DC voltage was applied to the DKDP to compensate for the phase mismatch. 
Figure 6 shows the OPA efficiencies obtained via DKDP versus rotation angle and compensation voltage. The rotation angle compensation ranges were approximately -0.03 to $+0.03^{\circ}$ and approximately -0.04 to $+0.04^{\circ}$ for $70 \%$-deuterated DKDP and $95 \%$-deuterated DKDP, respectively. Moreover, with the angle change of $0.01^{\circ}$, the compensation voltage values required for 70\%-deuterated DKDP and 95\%-deuterated DKDP were $\sim 3$ and $\sim 2.4 \mathrm{kV}$, respectively. The angle acceptance bandwidth (FWHM) of 70\%-deuterated DKDP and $95 \%$-deuterated DKDP can reach $\sim 0.1$ and $\sim 0.12^{\circ}$, respectively, which is $\sim 2$ times larger than that of OPA without compensation.
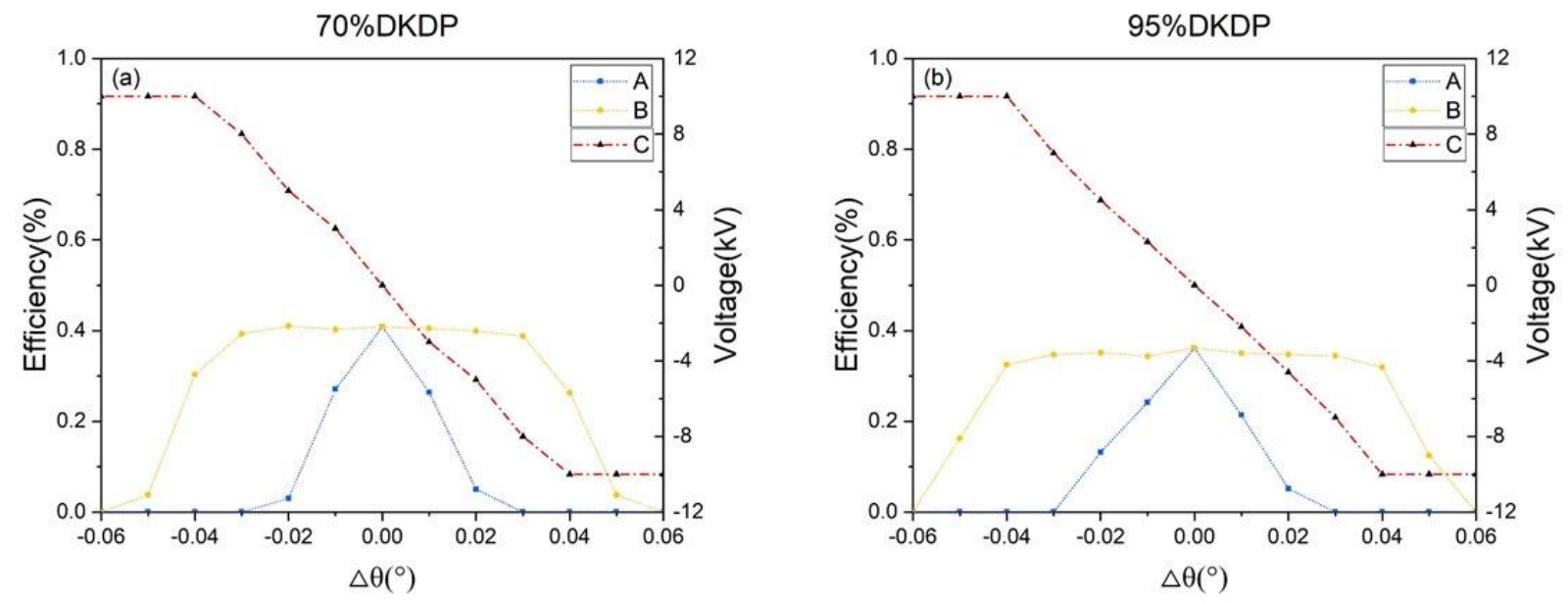

Figure 6. Experimental OPA efficiencies obtained via (a) 70\%- and (b) 95\%-deuterated DKDP versus angle and compensation voltage. Line A represents the OPA efficiency without compensation, Line B indicates OPA efficiency where the phase mismatch is compensated, and Line $\mathrm{C}$ represents the compensation voltage.

Figure 7 shows the perfect compensation voltage versus temperature and angle in the experiment. The $70 \%$-deuterated DKDP compensation voltage changed more evidently concerning temperature and angle. There are two main reasons: the refractive index of DKDP with a higher deuteration rate is less sensitive to temperature and angle changes; the electro-optic coefficient of DKDP with a higher deuteration rate is larger. Thus, the $70 \%$-deuterated DKDP compensation voltage slopes with respect to temperature and angle change were larger than those for the 95\%-deuterated DKDP.
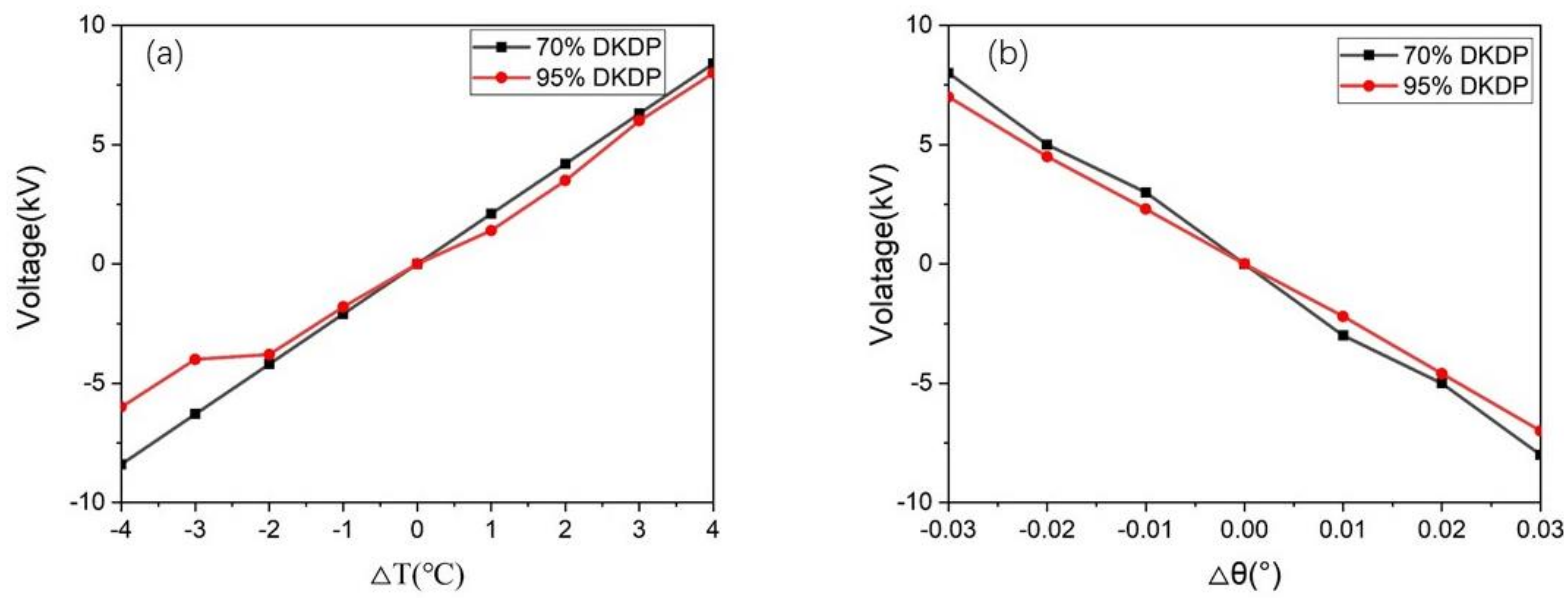

Figure 7. (a) Experimental phase mismatch compensation voltage versus temperature; (b) experimental phase mismatch compensation voltage versus angle. 


\section{Discussion}

Through the above analysis and experiments, we have proved that this method can compensate for the phase mismatch well. However, the temperature and angle compensation bandwidth are limited by the maximum value of the applied electric field. Therefore, a higher voltage value generator can achieve a wider temperature and angle acceptance bandwidth. The voltage applied to the crystal cannot be infinite, because excessive voltage will cause the crystal to be broken down, which also limits the compensation range of this method. This is especially obvious on small-diameter crystals. For large-diameter nonlinear crystals, a metal conductive coating can be added to the end face of the crystal. The uniform electric field will be generated after the voltage is applied. Moreover, electrode plate production is a relatively mature technology, and an external electrode plate can also be used to generate the electric field. The external electrode plate can also withstand a larger breakdown voltage, which is also more conducive to achieving a wider compensation bandwidth.

In the temperature-induced phase mismatch compensation experiment, the temperature inside the crystal was uniform. This method can compensate for phase mismatch very well. However, in some cases, the temperature in the crystal will not be uniform, and the compensation range will be further limited at this time. To overcome the impact of this situation, we can use multiple electrode plates to create different electric fields along the crystal to compensate for each part. Moreover, the phase mismatch compensation method can be widely used in optical parametric amplification systems such as BBO, DKDP, KDP, and LBO.

\section{Conclusions}

In summary, a method for phase mismatch compensation capable of improving the stability of OPA laser systems was described in this study. This method can accurately compensate for the phase mismatch caused by factors such as temperature changes and mechanical deformation (angle changes). The phase mismatch compensation characteristics via linear electro-optic effect in 70\%-deuterated DKDP and 95\%-deuterated DKDP were simulated. Temperature- and angle-insensitive OPA was demonstrated experimentally. The temperature range compensations of -4 to $+4{ }^{\circ} \mathrm{C}$ and -5 to $+5{ }^{\circ} \mathrm{C}$ were achieved for 70\%-deuterated DKDP and 95\%-deuterated DKDP, respectively. The temperature acceptance bandwidths (FWHM) of 70\%-deuterated DKDP and 95\%-deuterated DKDP were significantly widened. The rotating angle range compensations of -0.03 to $+0.03^{\circ}$ and $-0.04-+0.04^{\circ}$ were achieved for $70 \%$-deuterated DKDP and $95 \%$-deuterated DKDP, respectively. The angle acceptance bandwidth (FWHM) of 70\%-deuterated DKDP and 95\%-deuterated DKDP can be $\sim 2$ times larger than that of OPA without compensation.

In general, this method was effective in using the electro-optic effect to accurately compensate for the phase mismatch in OPA systems. This technology has a significant application in high-repetition and high-power OPCPA laser systems.

Author Contributions: D.L. designed and supervised the research; S.Y. performed the research and wrote the paper. All authors read and approved the manuscript.

Funding: This research was funded by the Shanghai Sailing Program (18YF1425900); National Natural Science Foundation of China (11774364); Bureau of International Cooperation, Chinese Academy of Sciences (181231KYSB20170022); and Strategic Priority Research Program of Chinese Academy of Sciences (Grant No. XDA25020302).

Informed Consent Statement: Informed consent was obtained from all subjects involved in the study.

Data Availability Statement: The available data has been stated in the article.

Conflicts of Interest: The authors declare no conflict of interest. 


\section{References}

1. Ross, I.N.; Matousek, P.; Towrie, M.; Langley, A.J.; Collier, J.L. The prospects for ultrashort pulse duration and ultrahigh intensity using optical parametric chirped pulse amplifiers. Opt. Commun. 1997, 144, 125-133. [CrossRef]

2. Bromage, J.; Bahk, S.W.; Begishev, I.A.; Dorrer, C.; Guardalben, M.J.; Hoffman, B.N.; Oliver, J.B.; Roides, R.G.; Schiesser, E.M.; Shoup, M.J., III; et al. Technology development for ultraintense all-opcpa systems. High Power Laser Sci. Eng. 2019, 7, e4. [CrossRef]

3. Rimantas, B.; Tomas, S.; Jonas, A.; Aidas, A.; Gediminas, V.; Darius, G.; Stanislovas, B.; Andrejus, M.; Arūnas, V. 53 W average power CEP-stabilized OPCPA system delivering $5.5 \mathrm{TW}$ few cycle pulses at $1 \mathrm{kHz}$ repetition rate. Opt. Express 2017, 25, 5797-5806.

4. Zhu, J.Q.; Xie, X.L.; Yang, Q.W.; Kang, J.; Zhu, H.D.; Guo, A.L.; Zhu, P.; Gao, Q.; Liu, Z.G.; Fan, Q.T.; et al. Introduction to SG-II 5 PW laser facility. In Proceedings of the 2016 Conference on Lasers and Electro-Optics (CLEO), San Jose, CA, USA, 5-10 June 2016; IEEE: Piscataway Township, NJ, USA, 2016.

5. Galletti, M.; Oliveira, P.; Galimberti, M.; Ahmad, M.; Archipovaite, G.; Booth, N.; Dilworth, E.; Frackiewic, A.; Winstone, T.; Musgrave, I.; et al. Ultra-broadband all-OPCPA petawatt facility fully based on LBO. High Power Laser Sci. Eng. 2020, 8, E31. [CrossRef]

6. Galletti, M.; Pires, H.; Hariton, V.; Alves, J.; Oliveira, P.; Galimberti, M.; Figueira, G. Ultra-broadband near-infrared NOPAs based on the nonlinear crystals BiBO and YCOB. High Power Laser Sci. Eng. 2020, 8, E29. [CrossRef]

7. Yu, L.; Liang, X.; Xu, L.; Li, W.; Peng, C.; Hu, Z.; Wang, C.; Lu, X.; Chu, Y.; Gan, Z. Optimization for high-energy and high-efficiency broadband optical parametric chirped-pulse amplification in LBO near $800 \mathrm{~nm}$. Opt. Lett. 2015, 40, 3412. [CrossRef] [PubMed]

8. Xu, L.; Yu, L.; Liang, X.; Chu, Y.; Xu, Z. High-energy noncollinear optical parametric-chirped pulse amplification in LBO at 800 nm. Opt. Lett. 2013, 38, 4837. [CrossRef] [PubMed]

9. Christoph, S.; Izhar, A.; Sandro, K.; Christoph, W.; Sergei, A.T.; Zsuzsanna, M.; Ferenc, K.; Stefan, K. Broadband amplification by picosecond OPCPA in DKDP pumped at $515 \mathrm{~nm}$. Opt. Express. 2012, 20, 4619-4629.

10. Marco, G.; Cristina, H.G.; Ian, M.; Ian, R.; Trevor, W. Influence of deuteration level of KD*P crystal on multi-PW class OPCPA laser. Opt. Commun. 2013, 309, 80-84.

11. Lozhkarev, V.V.; Freidman, G.I.; Ginzburg, V.N.; Katin, E.V.; Khazanov, E.A.; Kirsanov, A.V.; Luchinin, G.A.; Mal'shakov, A.N.; Martyanov, M.A.; Palashov, O.V.; et al. Compact 0.56 Petawatt laser system based on optical parametric chirped pulse amplification in KD*P crystals. Laser. Phys. Lett. 2007, 4, 421. [CrossRef]

12. Cai, X.; Lin, X.; Li, G.; Lu, J.; Hu, Z.; Zheng, G. Rapid growth and properties of large-aperture $98 \%$-deuterated DKDP crystals. High Power Laser Sci. Eng. 2019, 7, E46. [CrossRef]

13. Liang, X.; Xie, X.L.; Zhang, C.; Kang, J.; Yang, Q.W.; Zhu, P.; Guo, A.L.; Zhu, H.D.; Yang, S.H.; Cui, Z.R.; et al. Broadband main OPCPA amplifier at $808 \mathrm{~nm}$ wavelength in high deuterated DKDP crystals. Opt. Lett. 2018, 43, 5713-5716. [CrossRef] [PubMed]

14. Cui, Z.; Liu, D.; Sun, M.; Miao, J.; Zhu, J.Q. Compensation method for temperature-induced phase mismatch during frequency conversion in high-power laser systems. J. Opt. Soc. Am. B 2016, 33, 525-534. [CrossRef]

15. Sun, Z.; Cui, Z.; Sun, M.; Yuan, Y.; Li, Q.; Liu, D.; Zhu, J. Electro-optic coefficient measurement of a $\mathrm{K}\left(\mathrm{H}_{1-x} \mathrm{D}_{\mathrm{x}}\right)_{2} \mathrm{PO}_{4}$ crystal based on $\chi^{(2)}$ nonlinear optical technology. Opt. Express 2021, 29, 2647-2657. [CrossRef] [PubMed]

16. Yariv, A.; Yeh, P. Photonics: Optical Electronics in Modern Communications, 6th ed.; Oxford University: Oxford, UK, 2007.

17. Nikogosyan, D.N. Nonlinear Optical Crystals: A Complete Survey; Springer: Berlin/Heidelberg, Germany, 2005. 